\title{
[ 405 ]
}

\section{V.}

Befchreibung einer vulkanifchen Eruption nalue bei der Infel St. Michael (einer der Azoren),

\section{von}

S. TIrrAnd, Kapitain bei der Engl. Marine ").

Mit Zufitzen von Dr. Horner in Zürich.

Den 12. Juni 1812, als wir auf dem Schiffe Ia Sabrina der Infel St. Michael uns näherten, lahen wir zwei bis drei hauchläulen fich.erheben, die wir, der Aelnnlichkeit wegen, einem entfernten Gelechte zweier Kriegsl'chiffe zulchrieben. Allein der Hauch nahm immer mehr zu, und gewann bald eine weir grülsere Ausdehnung, als mit jener Urfache verträglich war. Wir erinnerten uns aun, bei unfrer Abreile von Liđabon gehürt zu haben, dals im letzten Januar oder Febr. nahe bei der Inlel St. Michael ein Vulkan fich gezeigt habe, und wir glanbten allo diel'en Kauch für einen Ausbruch deffelben halten zu mülfen. Allein auch diefe Vorausfetzung wurde am andern Morgen, als wir bei Ponta del Gada

9) Mitgetheilt von Sir Jor. Banks für die Philoj: Transact. for 1812, und ron da in dio Bibl. brit. Nn. 404. überragen. 


\section{[ 406$]$}

vor Anker gingen, durch den Bericht der Einwohner widerlegt, deu zu Folge der Ausbruch voun Januar lich bald gelegt, und die von uns wahrgenommene Erfcheinung erft leit zwei Tagen, eine Seerneile (a league) von der Stelle der frühern Eruption entferut, begonnen habe.

Es log mir lehr am Herzen, diefen fonderbaren Kampf zweicr fo mächtigen Elemente etwas nüher zu betrachten, und ich brach daher gleich den fulgenden Morgen (den 1. (ten) von der Stadt Ponca del Gada auf, in Begleitung des Generalcoululs der Azorilchen Inleln, Herrn Read, und zweier feiner Freunde. Nach einem Marlich von etwa 20 engl. Meilen quer durch das nordüftliche Ende der Inlel gelangten wir an deu Rand cines ungefähr yoo Fufs hohen, faft lenkrecht abfallenden Vorgebirges, deffen Fuls nur eine Engl. Meile vom Vulkan entfernt war. Und hier erblickten wir plützlich das ganze Schaulpiel in leiner fürchterlichen Grülse vor' uns. Die folgende Befchreibung giebt davon nur ein fehr unvollkommnes Gemälde.

Man denke lich eine ungeheure Rauchmafle, aus dem Meere fich erhebend, deflen filberfarbene Flüche ein kühler Wind in leichte Furchen zertheilte. War die Mafie in Kuhe, lo Iclien eine kreisfürmige Wolke auf dem Walfer ausgelireitet zu reyn, wie ein grolses horizontales Rad, das der Wind allmählig in weite Falten zerlegte. Plützlich ftieg dann eine fchwarze Süule von Alche und Schlacken enpor, die wie ein lchieflichender 


\section{[ 407 ]}

Thurm fich so bis 20 Grade vom W'inde abwärts neigte. Bald erhob lich eine zweite, eine dritte; eine vierte Säule, die alle fich über einander bis zur doppelten Höhe unfers Standpunktes aufthürm: ten. Wenn die Heftigkeit der aufteigenden' Bewegung nachliefs, zertheilte lich die Wolkenmalfe in mehrere Zwveige, welche grolsen Bülchen vereinigter Tannen glichen, fich aber bald in lehr elegante Winclungen eines weifsen Rauches verwandelten. lis fehwamnen in ihr cine Menge Afchentheilclen; zu gewiffen Zeiten glichen fie einem Haufen weifser und fchwarzer in den Wind geworfener Straulsfedern, und zu andern Zeiten den hängenden Zweigen der Trauerweide. .

Während dielem Ausbruche gingen aus der lichteften Stelle der vulkanifchen Wolke lebhafte Blitze hervor, und die Rauchmaffe, welche fich nun weit iiber jene Alchenauswürte erhob, zertheilte fich in ungeheure Flocken, die der Wind horizontal vor fich hertrieb, und die hier und da Waflerhofen aus dem Meere zu fich aufzogen, durch welche diefes grolse Schaufpiel noch feltfamer und prachtvoller wurde.

Die Stelle des Meeres, wo leit vier Tagen die Eruption fich gezeigt hatte, war mehr als 30 Faden tief. Bald, nachdem wir an den Rand des Abhanges, auf dem wir ftanden, hinausgetreten waren, behuuptete ein Bauer, er Iähe eine Bergf pitze 'aus dem Waffer fich erheben. Wir konnten nichts davon entdecken; allein in weniger als $\frac{x}{2}$ 


\section{[ 108$]$}

Stunde wurde diefe Spitze vollkommen fichtbar, und elie wir den Platz verliefsen, das heilst 3 Stunden nach. unfrer Ankunft, erblickten wir einen fürmlichen Krater über dem Meere. Er hatte nicht weniger als zo Fuls Hïhte auf der Seite, wo die meilie Afrhe tiel; lein Durchmeffer mochte 400 bis 500 Fuls betragen.

Die grolsen Ausbrïche waren meiftentheils von Detonationen begleitet, die dem vermilchten Getüfe eines ununterbrochenen Kanonen - und Gcwehrfeuers glichen. Meine Begleiter wollten auch einige leichte Erdftüfse verfpürt haben, die ich jedoch nicht empfunden hatte, und auf Rechnung ihrer Einbildung l'chrieb. Allein, während wir in einer Entfernung von 5 bis 6 Ellen vom Rand des Ablanges lafsen und afsen, lahen wir plützlich eine der lchünften Eruptionen aus dem Krater herauftchiefsen, und verlipürten zu gleicher Zeit einen fehr fụ̈hllbaren Erdfiols. „Dieles Mal galts! “6 riet jeder von uns, indem wir lchnell auffprangen; und kaum hatten wir diefe Worte ausgefprochen, als etwa 50 Ellen von uns zur Linken ein beträchtliches Stiick des Abhanges fich losrifs, und mit lautem Krachen ins Meer fiürzts. Wir zogen uns nun einige Klafter weiter zurïck, und letzten unlere Mahlzeit fort.

Tags daraut, den 15. Juni, hatte ich den Generalconful an Bord. Ich lichtete die Anker, und nïherte mich dem Vulkan, um ihn in der Nacht zu beobachten. Allein gegen n:eine Erwartung 


\section{$\left[\begin{array}{lll}4 & 49\end{array}\right]$}

wurde dar Wind frifcher, das Wetter neblich, und der Vulkan viel ftiller als atI vorigen Abend. Selten [chleuderte er Blitze aus, und liicls nur von Zeit z.u 'Zeit Flammenfirüne hèrvor, denen :ilnnlich, welche man zuweilen atit den Schorufteinen von Glashiutten oiler Sclunnelzijlen wahrniumnt.

Als wir genall unter der grofsen lauchwolke durchliegelte-n, ungefilir 3 bis 4 Meilen rom Vulkan, wurde das Verdectk unlers Schilfs mit fcluwarzor und feiner Afche bedeckt, die mit einigen Regentropten herunterlial. Am Mors n berignen wir uns wieder auf unfion vorigen Ankerplatz, und Taiss darauf verlieis ich St. Michael, um weiter zu kreutzen. In der Nacht vom 1 Gten lilien wir noch sin pnar Eruptionen des Vulkans. Die Flanime war azhaltend und felir leblaft; allein wir waren indilr als zo Meilen davon entfernt.

Als ich am 4. Juli wieder in diele Gegend kam; erlaubte mir der Wind, leelır nahe bei dem Vulkan vorbei zu gelien. Er war nun eine fürnliche Inlel geworden, deren Mitte lich melir als 80 Ellen ïber das-Mcer erhob. Alles war jetzt ruligy, und ich bekam Luit, ihn in Beggletitung von eimicen Oilicieren zn befuchen. Als wir in Boote nïher kame:ı, bemerkten wir, dals der Boden noch dampftc. Wir errcichten ungenthlotet der liarken Btandungs das titer ohne lunderlinhe Getalir, und fanden cinen fchmalen Strich von lchwarzer Afche, von dein an der lBoden fich me:ilt lis liteil erhob, dal's es unnï̈glicil war. hinant za klettern; und dia, wo diefies 


\section{[ f́ro]}

zur Noth noch möglich gewelen wäre, war die Errle viel zu heils, als dals man Gich hüher als einige Fadeqn hätte hinauf wagen dürfen. Der Abhang ging eben fo fteil in das Meer hinab, und die Sonde gab 20 bis 50 Ellen rom U'ier fchon 25 Faden Tiefe. Ick brauchte 12 Minuten, uin rund um die Infel z1z gehen, und fchätze ilıren Umfang auf 'nahe eine Meile. Das merkwiirdigfie war der Krater, dellien eigentlicher Schlund fich fo zienlich in der WalCerhühe des Meeres beland. Diele Fü̈hlung war damals mit koctsendem Wafler angefitlt; ein kleiner Bach von etwa 6 Ellen Breite fülirte das Walfer aus derfelben $a b$, und erfïllte fie wiedler mit lieigender Fluth; es war ganz nalie am Heere noch fo heifs, dafs ich dic Fland nicht darin zu halten und kaum den Finger einen Augenblick hinein zu tauchen vermochte.

Die ganze Ceftaltung dieles Thuils der Inlöl zeigte offenbar, dal's wihrenul der Eruptionen das Meer an zwei Stellen in den Krater eingedrungen war. Denn die üfiliche Seite des kleinen Bachs war von einem 20 bis 30 Fuls hohen Ufer Legrinzr, welches eine Hallinfel ron eben fo viel Breite und 5o bis 6o Fuls Lünge bildete, die mit dem Reft der Infel durch eine Ichmale, etwa 5o Fufs lange, aus Lava und vulkanilcher Afche gebildete Erdzunge verbunden war, an deren Ende der Krater fich amphitheatralifch erhob.

Aus einer Entfornung von 2 Lis 3 Meilen wiirde man diefe Inlei fiir ein Werk der Kunlt, 


\section{$\left[\begin{array}{lll}4 & 4 x\end{array}\right]$}

fiir ein Fort oder eine Schanze gehalten haben. Uniiberfteigliche Findernifle machten es uns unmïglich, den Gipfel zu erreichen. Er war nun von der Seite der Landenge zugänglich, und felbft hiex erhob er fich [o fteil, dals wir nur mit Hïlfe eines in die Ercle gefteckten Ruders ilın erlliegen; und kaum hatten wir ihre Hüho erreicht, fo hielt uns eine neue Schwierigkeit auf. Es war unmüglich, nur einen Schritt weiter zı gehen, denn die entgegengeletzte Seite liel faft lenkrecht ab. Die Kante, auf der wir uns befanden, glich dem Giebel eines Daches. Wir hielten uns auf ihr reitend mit den IÏ̈nden teft, bis wir endlich eine Stelle fanclen, wo man aufrecht flehen konnte. Hier pflanzten wir eine Fahne aut, als ein Zeichen der Befitznehmung diefer unter unfern Augen entftandenen Infel. Am Fuls der Stange vergruben wir eine wohl verfiegelte Bouteille, welche die vollfiändige Erzählung diefer Begebenheit und den Namen Sabrina, den wir der Inlel gegeben hatten, enthielt.

Ich hatte im Krater das volltändige Skelett eines grolsen Filches gefunden; allein die Knochen waren durch die Hitze fo verzehrt, dafs fie beim Wegnehmen in Stiicken lielen. Nach Auslage der Einwohner auf St. Michael fand fich auch beim Anfang der Eruption in allen benachbarten Buchten eine grolse Menge todter Fifche, die walurfcheinlich ein $O$ pfer der grofsen Temperatur-Verinderung ihres Elements geworilen waren. 


\section{[ 412 ]}

Diefe Infel beftelıt, wic die meiften Gegenden vulkanil'chen Urlprungs, hauptlächlich aus porijlen Sublianzen, meiftens im Zuftande von Alche oder Pozzolan-Erde. Man lindet hier und da fteinartige Mafren, die mir ein Cemifch von Kalkerde und Eifenminer zu leyn lchienen; wortiber jedoch die nitgebrachten Exemplare don beften Auflchluls geben werden.

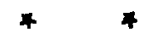

Die vorftelicnde befchreibung eines Naturprozelles, der felten lis willfiändig beobichtet werden kann, führt mir eine Erfcheinung in das Gediclıtnifs, die wir auf der Krnfenfernllichen lheile un die Welt, und zwar ebenfills in allantifchen Nleere, walirycnommen haben, und die mit dem Anfange dieler Begebenheit viel Achnlichos hat. Den 1 gten Mhai 1806, Abends non 5! Uihr, erblickte man in Wivil, bei feldr liellem Wetter und wolkenlufem Ilimmel, etwas wic Runch am Horizont. Er glich

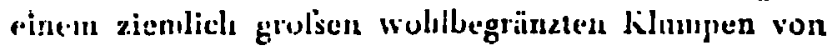
gleicher Höhe und Durchmeffer, und mochte, alswedifelnd entfichend und verfolwindend, eine Vierat.limude gedauert haben. Die Matrofen, welche es zuterli wallingenosumen hatten. zeigten es $z u f_{p}$ it an, fo dal's wir es nur kurz ror feinem Verfehwsinden falhen; wodurch anch eine genatuere Mefiung unmöglich wurde. Niach meine'm L'rtheil war es weniofitens 2, mah andern 5 bis f leutfche Mleilen entfernt, und

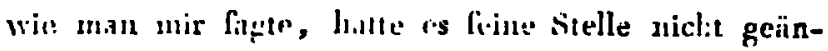
dert. linige rirthen anf ein emterntes Gelechr, in-

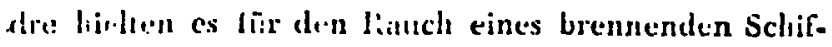
lis; aldein die hurse Laber des lhänomens, fo wie 


\section{[ $\quad 413$ ]}

der Umfiand, dafs gar keine Explofion gehört wurde, fchien beiden Vermuthungen 20 wider [precben, und es blieb uns nur tibrig, es für die Wirkung einer val-' kanichen Aufwallung unter Wafler zu halten; was in jener Meeresgegend bei den vulkanifchen Infeln St. Helena und Afcenfion, und in der Näbe der ziemlich ungewiffen Infel St. Panl nicht eben unwahrfcheinlicly war. Unfere Beftimmung erlaubte uns nicht, diefer vorübergebenden krfcheinung weiter nachzugehen; vielleicht dafs fpäterhin an eben diefer Stelle *) eino Infel oder Untiefe zum Vorfchein kömmt, wo niemand vorher etwas wahrgenommen hatte.

Noch immerfort werden in Mecrestitichen, dio von unzähligen Schiffen durchfclunitten werden, of beinahe mitten in den Hauptftrefsen im freien Ocean, ifolite Felfen, Piks, Untiefen u. dgl. éntdeckt, die, wenn auch die Nacht oder der mit ithrer Kntdeckung oft verbundne Untergang des Scliffes fie unfrer Renntnifs lange entzichen mag; doch lingft hatten gefunden werden müfrn, weun Ge nicht netuern Urfprungs wirren. Die Ungewif́sheir, die über die grofse Menge der Cintiefen (Shoals, Vigies), on denen das atlantifche Meer, to wie die vulkanifche Gegend der Sìdfeeinfeln vorzüglich reich ift, und felblt ïber kleinere, nicht mehr aufzufindende Infeln obwaltet, möchte oft wohl eben fo felur auf Rechnung vulkanifclier Bildungen umd Zerfiürungen durch Fluthen oder Fìuer kommen, als einer eingebildeten Wahrnehrnung oder unrichtigen Ortsbeflimmung der Seefalirer zumafchreiben feyn. Und diefe fortdanernde Schüpfung möchte der Vollkommenheit unfrer nautifchen Geographie axch in nürdlichen Meeren eben fo hinderlich fejn, als es die Bildung der Korallenbänke in der Sũdfee iff.

7) Sie liegt in $2^{\circ} 35^{\prime} \mathrm{S}$. und $20^{\circ} 45^{\circ} W$. v. Greenwr. $H$. Annal. d. Phylit B.42. St. 4. J. 1812. St 12. E. 


\section{[ $4: 1$ ]}

Hr. Dr. Langsd orf hat von Seiner Reife nach der Nordwefiküfte Amerika's eine ähnliche, doch ziernlich unvollkommne Erzäblung ron der Bildung eimer vulkanifckien Infel wefilich von Unalaska, und nordnordweflich von der Infel (Umnak *) mitgeloracht. In der Nahe cines dort ftehenden Felfens, auf weelchein die Alenten von den älteften Zeiten her Seelöwen und Seehunde erlegt hatten, zeigte fich 1795 ein Nebel, welcher fillti bei dem helleften Wetter ftehen blieb, und ein Einwoliner ron Unalaska, welcher endlich dahin fuhr, kam mit der Nachricht zurück, das Mecr koche dafellit. Der Rauch dauerte olne grofse Abänderung bis zum J. 1800 fort, und nun erblickten die erfinunten In. fuluner eine kleine nie gefelhene Infel in der NichbarSchaft jenes Felfen. Lis war ein Pik, der unaulhürlich Raucli und Flammen ausfipie, der mir zur Zeit eines ftarken Erdbebens auf L'nalaska niclst brannte, withrend deTen der Vulkan auf Unalaska wũthere. Linigo Infinlaner, welche im April 1806 die neue Infel belischit hátten, fagten, fie häıten fie in etwa 6 Stundest unrudert, welehes für ihren Lrnfang etwa 30 Werlte giebt. Der Berg war zu erfteigen viel $z$ fieil, fonft würden lie, wie fie glinubten, feine Spitze vohl in 6 stumlen crrcicht haben. An der fieilen Nurdleite Huls buftïudiig eine weiche Mattie vom Gipfel herunter in das Meer, und die Erde war dort zum Landen zu heifs. Sie fliegen dalier an flachern Sïduier aus, und gel.sggitin nit grofser Befchwerlichlicit niclit iüllig bis zur haiben Hölse des Piks, no die Erdes meshiich wärmer was, und aus mehrern Hühlen des Burge:s liauch und i lizze leervurdrang. Mlit cinem Erfumnen, dis fie felbli beim Wiedererziblen noch init lizeurde corfiillic, fanden tie Serelüwen.

; Verg! oten S. 21 :. 


\section{$\left[\begin{array}{lll}415 & ]\end{array}\right.$}

Alifch, welches fie beim Ausruhen an' Stöcken in diefo Löcher hineingehängt hatten, fehr gut gebraten. Mangel an Walfer, das fie auf der Infel zu finden obne Urfacho gehofft hatten, nöthigte fie bald zar Rũckkehr Aufser einigen Stücken gediegenen Schwefels brachten fie nichts mit von da zurück. Dio Steine, lagten fie, wären wie die auf Unalaska.

Horner.

\section{VI. \\ Mesmer's thierifcher Magnetismus.}

„Die Gefchichte lehrt, (agt. Hr. Prof. Link *), dals unter den Naturforlehern: die Aerzte es vorzüglich waren, welche jedem neuen plitofophifchen Syfteme huldigton. Sie haben es mit dem felwwerfen Theile dex Naturkunde. en thun, yon dem wir eigentlich noch nichts wiflen; fie mülten fich ihrer Kunft wegen das Anfelin geben, als wüfsten fie etwas. Daher der Beifall, den fie den philofophifchen Syftemen [und den eufserordentlichen Dingen] defto,lieber zollen, , je weniger. fe folche verftehn."

Bekanntlich hat man in den neuften Jahren in einigen Theilen Deutfchlands auch au Mlosmer's thiorifchem Magnetismus gegriffen, um ärztlichen Schulen einen Ichimmernden Glanz zu geben. Eine Wiedererinnerung an die kritifchen Verhandlungen über den Mesmerianismus in Paris, zu Mesmer's glänzender Zeit, welche fich in den (ranzöfifchen Blättera *t) findet, Ee 2

7) In Reinem Tchäubaren Werke: Uaber NaturphiloTaphie. Roltock 1806 .

c.

") Durch Hra. Montegro in dem Journal de Paris, boi Golegenhoit der Anzeige eines Werks über den Magnecismus, und daraus in dom Monkew rom 19. Nor. 18 sa. $O$. 